Research Paper

\title{
Resistance training and redox homeostasis: Correlation with age- associated genomic changes
}

\author{
Ivan Dimauro ${ }^{a, *}$, Mattia Scalabrin ${ }^{a}$, Cristina Fantini ${ }^{a}$, Elisa Grazioli ${ }^{a}$, \\ Maria Reyes Beltran Valls ${ }^{a}$, Neri Mercatelli ${ }^{a}$, Attilio Parisi ${ }^{\mathrm{b}}$, Stefania Sabatini ${ }^{\mathrm{a}}$, \\ Luigi Di Luigi $^{\text {c,1 }}$, Daniela Caporossi ${ }^{\mathrm{a}, 1}$ \\ a Unit of Biology, Genetics and Biochemistry, Department of Movement, Human and Health Sciences, University of Rome "Foro Italico", Piazza Lauro de Bosis \\ 15, 00135 Rome, Italy \\ ${ }^{\mathrm{b}}$ Unit of Sport Medicine, Department of Movement, Human and Health Sciences, University of Rome "Foro Italico", Rome, Italy \\ ' Unit of Endocrinology, Department of Movement, Human and Health Sciences, University of Rome "Foro Italico", Rome, Italy
}

\section{A R T I C L E I N F O}

\section{Article history:}

Received 1 August 2016

Received in revised form

19 September 2016

Accepted 20 September 2016

Available online 21 September 2016

Keywords:

Telomeres

DNA methylation

Myeloperoxidase

TrxR1

MnSOD

\begin{abstract}
A B S T R A C T
Regular physical activity is effective as prevention and treatment for different chronic conditions related to the ageing processes. In fact, a sedentary lifestyle has been linked to a worsening of cellular ageing biomarkers such as telomere length (TL) and/or specific epigenetic changes (e.g. DNA methylation), with increase of the propensity to aging-related diseases and premature death.

Extending our previous findings, we aimed to test the hypothesis that 12 weeks of low frequency, moderate intensity, explosive-type resistance training (EMRT) may attenuate age-associated genomic changes. To this aim, TL, global DNA methylation, TRF2, Ku80, SIRT1, SIRT2 and global protein acetylation, as well as other proteins involved in apoptotic pathway (Bcl-2, Bax and Caspase-3), antioxidant response (TrxR1 and MnSOD) and oxidative damage (myeloperoxidase) were evaluated before and after EMRT in whole blood or peripheral mononuclear cells (PBMCs) of elderly subjects.

Our findings confirm the potential of EMRT to induce an adaptive change in the antioxidant protein systems at systemic level and suggest a putative role of resistance training in the reduction of global DNA methylation. Moreover, we observed that EMRT counteracts the telomeres' shortening in a manner that proved to be directly correlated with the amelioration of redox homeostasis and efficacy of training regime, evaluated as improvement of both muscle's power/strength and functional parameters.

(c) 2016 The Authors. Published by Elsevier B.V. This is an open access article under the CC BY-NC-ND
\end{abstract} license (http://creativecommons.org/licenses/by-nc-nd/4.0/).

\section{Introduction}

With increasing age, genetic vulnerabilities, underlying diseases, and physiological impairments increase the risk for decline in physical functioning [50]. Despite it is known that physical activity (PA) prevents functional decline among older people, with a positive influence on health and survival compared with a sedentary lifestyle, inactivity continues to be a major public health problem $[70,14,5]$. Actually, a sedentary lifestyle has been linked to a worsening of cellular ageing biomarker such as telomere length

\footnotetext{
* Corresponding author

E-mail addresses: ivan.dimauro@uniroma4.it (I. Dimauro), mattia.scalabrin@yahoo.it (M. Scalabrin), cristina.fantini@uniroma4.it (C. Fantini), elisa.grazioli@uniroma4.it (E. Grazioli), reyes.bel@outlook.com (M.R. Beltran Valls), neri.mercatelli@uniroma4.it (N. Mercatelli), attilio.parisi@uniroma4.it (A. Parisi), stefania.sabatini@uniroma4.it (S. Sabatini), luigi.diluigi@uniroma4.it (L. Di Luigi), daniela.caporossi@uniroma4.it (D. Caporossi).

${ }^{1}$ These authors contributed equally to this research.
}

(TL) in white-blood-cell [14], with increase of the propensity to aging-related diseases and premature death [50]. Telomeres are repetitive sequences of DNA (5'-TTAGGG ${ }_{n}-3$ ') located at the ends of mammalian chromosomes. They decrease in length with age, reducing the stability and function of chromosomes [19]. Besides the end-replication problem, telomere-specific DNA damage induced by oxidative stress has been extensively studied as a factor that may contribute to telomere shortening [29]. TL has been indeed reported to predict cancer, mortality and cardiovascular events [36], where chronic oxidative stress seems to play a major role in the pathophysiology of chronic inflammation. Moreover, clinical data suggest that parameters of telomere biology in circulating cells can be used as indicators of the effect of therapeutic intervention for cardiovascular morbidity [21].

Circulating blood leukocytes represent a resource in telomere biology because of the high correlation between TL in these cells and those of other tissue types [25,72]. However, it must be considered that $\mathrm{TL}$ in different cell types may better reflect specific diseases, tissue-specific aging, or cell-specific adaptations [67]. To 
date, there are many evidences about the association between habitual PA and longer leukocyte telomere length (LTL) $[14,17,37,40,71,18,57,39]$, but most of them derive from studies where aerobic endurance-exercise training was used as intervention [14,17,37,18,57]. Moreover, they have shown conflicting results, with telomeres that can take three different trajectories (expected shortening, maintenance, and lengthening). Nevertheless, little is known about the effect of resistance training on telomere length, so far. Resistance training has the potential to counteract the age-related decline of muscle strength, power output, and muscle mass in elderly populations. In fact, the magnitude of these adaptations seems to be similar to those observed in untrained young subjects $[45,51]$. Therefore, this type of training has clinical relevance due to its benefits on the neuromuscular system and on the daily living activities performance [5].

We recently showed that 12 weeks of low frequency, moderate intensity, explosive-type resistance training (EMRT) could be proposed as an effective exercise intervention for improving the overall health of older people. Indeed, EMRT not only enhanced the muscle strength, power, and functional performance without detrimental effects on cardiovascular and inflammatory parameters [6], but improved the general adaptive response to oxidative stress related to intense acute exercise [11].

Given the extensive evidence that aging can impact the genome modifying its structure-function relationship through epigenetic changing (i.e. DNA methylation) and alter telomere dynamics [30], the aim of the present study was to test the hypothesis that EMRT may attenuate the age-associated genomic changes. In particular, LTL, global DNA methylation and global protein acetylation, as well as the expression of TRF2, Ku80, SIRT1, SIRT2 and proteins involved in the apoptotic pathway (Bcl-2, Bax and Caspase-3) and the antioxidant response (TrxR1 and MnSOD) were evaluated before and after EMRT in peripheral blood mononuclear cells (PBMCs) of elderly subjects. Moreover, a correlation analysis was performed to verify whether LTL correlates with the markers of redox homeostasis or with the decline in functional parameters in ageing.

\section{Material and methods}

\subsection{Study design}

Recently we have conducted a controlled trial in which elderly subjects (70-75 years) were randomly divided into training and control group, to evaluate the effect of an EMRT protocol for 12 weeks on muscle strength, power and functional performance, as well as the adaptive response to oxidative stress induced by an acute exercise $[11,6]$. The study has been expanded utilizing subjects from both the trained group (TR: $n=10)$ and from the control group (CTRL: $n=10$ ), successfully able to complete the blood samplings at baseline and after the EMRT protocol period. Our subset resulted to be fully representative of the original groups, being the anthropometric, physiological and functional characteristics overlapping the values of the previous study at both baseline (PRE) and POST-EMRT training (Table 1) [6]. Physical activity level was evaluated using the Modified Baecke Questionnaire for Older Adults [69]. Diet composition and nutrient intake, adjusted for total calories, were similar among the groups (data not shown). No significant difference between groups was reported comparing baseline level of all parameters analyzed and characteristics of participants. Unless differently stated, no gender differences were found concerning all parameters analyzed.

Participants were seated comfortably for $10-15$ min prior to resting blood pressure and heart rate measurements. Further, height and weight were recorded during a clinical visit, from
Table 1

Baseline characteristics of participants.

\begin{tabular}{|c|c|c|}
\hline Characteristic & TRAINED $(n=10)$ & CONTROL $(n=10)$ \\
\hline Gender & 5 males 5 females & 5 males 5 females \\
\hline Age (years) & $72 \pm 1$ & $72 \pm 1$ \\
\hline Height $(\mathrm{cm})$ & $166 \pm 9$ & $167 \pm 10$ \\
\hline Body Mass (Kg) & $70 \pm 2$ & $72 \pm 3$ \\
\hline$B M I\left(K g / m^{2}\right)$ & $23 \pm 2$ & $25 \pm 1$ \\
\hline Systolic BP (mmHg) & $123 \pm 4$ & $131 \pm 4$ \\
\hline Diastolic BP (mmHg) & $78 \pm 3$ & $75 \pm 3$ \\
\hline Resting $H R\left(b \times \min ^{-1}\right)$ & $68 \pm 9$ & $69 \pm 8$ \\
\hline Physical activity level & $20 \pm 1$ & $19 \pm 2$ \\
\hline \multicolumn{3}{|c|}{ Modified Baecke Questionnaire (score) } \\
\hline Household & $14 \pm 1$ & $13 \pm 1$ \\
\hline Sports & $3 \pm 1$ & $4 \pm 1$ \\
\hline Leisure & $3 \pm 1$ & $2 \pm 1$ \\
\hline \multicolumn{3}{|l|}{ Muscle power $(\Delta \%)$} \\
\hline LegExt & $35.9 \pm 5.2^{\mathrm{a}, \mathrm{c}}$ & $-0.9 \pm 1.9$ \\
\hline CMJ & $17.5 \pm 4.3^{\mathrm{a}, \mathrm{c}}$ & $-2.0 \pm 2.8$ \\
\hline \multicolumn{3}{|l|}{ Muscle strength $(\Delta \%)$} \\
\hline LegExt & $16.4 \pm 1.5^{\mathrm{a}, \mathrm{c}}$ & $-0.3 \pm 1.5$ \\
\hline \multicolumn{3}{|l|}{ Functional tests $(\Delta \%)$} \\
\hline $6-m$ walking & $-9.0 \pm 1.6^{\mathrm{a}, \mathrm{c}}$ & $1.4 \pm 1.3$ \\
\hline 6-m walking loaded & $-10.0 \pm 2.1^{\mathrm{a}, \mathrm{c}}$ & $1.5 \pm 2.2$ \\
\hline Stair-climbing & $-8.0 \pm 3.1^{\mathrm{a}, \mathrm{b}}$ & $3.6 \pm 2.0$ \\
\hline Stair-climbing loaded & $-12.0 \pm 2.5^{\mathrm{a}, \mathrm{c}}$ & $-0.2 \pm 2.7$ \\
\hline
\end{tabular}

All values represent means + SEM. The delta percentage was calculated trough the standard formula: $\Delta \%=[($ POST-test score-PRE-test score $) /$ PRE-test score $] \times 100$. BMI (body mass index); BP (blood pressure); $\mathbf{m m H g}$ (millimeters of mercury); LegExt, leg-extention; CMJ, countermovement jump.

a $\mathrm{p}$ values $<0.01$.

b $\mathrm{p}<0.05$ for changes within-group after the intervention period.

c $\mathrm{p}$ values $<0.01$ for changes between-group after the intervention period (TR vs. CTRL).

which BMI was calculated. Exclusion criteria included co-morbidities that could interfere with LTL analysis between active and inactive subjects (e.g., heart disease, heart attack, stroke, diabetes mellitus types 1 or 2, osteoarthritis of the hip or knee, Parkinson disease, cancer, psychological chronic stress or any other mood disorders). All participants were fully informed of the research design and associated benefits and risks of the investigation before signing an informed consent form approved by the relevant Ethics Committee.

\subsection{Blood sampling and PBMCs isolation}

Before and after 12 weeks of intervention, fasted blood samples were drawn from the antecubital vein while subjects remained in reclined position. Samples in additive-free tubes (BD Biosciences, San Jose, CA, USA) were left at room temperature for coagulation for at least $1 \mathrm{~h}$ and then centrifuged $(2,500 \mathrm{rpm} \times 10 \mathrm{~min})$ for serum separation. Blood sampled in EDTA tubes (BD Biosciences) were used for plasma collection by centrifugation of whole blood $\left(2,500 \mathrm{rpm} \times 10 \mathrm{~min}\right.$ at $\left.4{ }^{\circ} \mathrm{C}\right)$ and for PBMC isolation. Whole blood, serum, plasma, and PBMC samples were aliquoted and stored at $-80{ }^{\circ} \mathrm{C}$ for further analyses. PBMCs were isolated by density gradient centrifugation from each sample (Ficoll-Paque plus; Amersham Pharmacia Biotech, Piscataway, NJ).

\subsection{EMRT and functional parameters}

Detailed information about the explosive-type resistance training (EMRT) protocol and the evaluation of functional parameters has been published elsewhere [6]. Briefly, low frequency EMRT ( 2 days/week) for 12 weeks was employed. For each machine, the resistance was set at $70 \%$ of the one-repetition maximum (1RM), and subjects performed 3-4 sets of 10-12 
repetitions. Every two weeks, resistance was incremented to induce volitional fatigue in the $10-12$ repetition throughout the training program. The increase in resistance was usually of $2.5 \mathrm{~kg}$, and before the increment, the perceived fatigue was evaluated to consider also the individual perception of the load.

One repetition maximum (1RM) test was utilized to evaluate lower body extremity maximal strength (Leg extension-LegExt), whereas muscle power was evaluated during countermovement jump (CMJ). Stair climbing and 6-meter walking time tests, with or without one dumbbell in each hand with a total load corresponding to $12 \mathrm{~kg}$ for women and $16 \mathrm{~kg}$ for men, were performed to evaluate the functional capacity of each subject.

\subsection{DNA extraction and telomere length analysis}

DNA was extracted from whole blood using ChargeSwitch gDNA 50-100 $\mu \mathrm{L}$ Blood Kit (Invitrogen, Carlsbad, CA). All DNAs were extracted in the same assay in order to reduce variability in the recovery of DNA from different samples. The ratio of the absorbance at 260 and $280 \mathrm{~nm}$ (A260/280) was used to assess the purity of DNA. An absorbance ratio $1.7<$ A260/280 $<1.9$ was considered acceptable. The integrity of random DNA isolates was evaluated by agarose gel electrophoresis.

Measurement of relative telomere lengths (Telomere PCR to Single-copy gene PCR or T/S ratio) was determined by quantitative real-time polymerase chain reaction (RT-PCR) as described by Cawthon [10] with the following modifications. The forward primer for the telomere PCR (T PCR) was tel1b [5'-CGG TTT GTT TGG GTT TGG GTT TGG GTT TGG GTT TGG GTT-3']. The reverse primer was tel2b [5'-GGC TTG CCT TAC CCT TAC CCT TAC CCT TAC CCT TAC CCT-3']. The forward primer for the single-copy gene (S PCR) was Hbb3-F [5'-TGT GCT GGC CCA TCA CTT TG-3']. The reverse primer was Hbb4-R [5'-ACC AGC CAC CAC TTT CTG ATA GG-3']. Tubes containing $150,75,37.5,18.75$, and 9.4 ng of reference DNA were included in each PCR assay to determine the standard curve. The telomere reaction mixture consisted of $15 \mathrm{ng}$ genomic DNA, 1X SYBR Green PCR Master Mix (Applied Biosystems Inc.), $2.5 \mathrm{mM}$ DTT, $100 \mathrm{nM}$ Tel1b primers and $900 \mathrm{nM}$ Tel.2b primer. The assay was performed using the 7500 Real-Time PCR System (Applied Biosystems Inc.). The T PCR thermal cycling profile consisted of for 1 cycle at $95{ }^{\circ} \mathrm{C}$ for $10 \mathrm{~min}, 30$ cycles of $95^{\circ} \mathrm{C}, 15 \mathrm{~s}$, and $54^{\circ} \mathrm{C}$, $1 \mathrm{~min}$. The S PCR thermal cycling profile consisted of for 1 cycle at $95^{\circ} \mathrm{C}$ for $10 \mathrm{~min}$, followed by 40 cycles at $95^{\circ} \mathrm{C}$ for $15 \mathrm{~s}, 58{ }^{\circ} \mathrm{C}$ for $20 \mathrm{~s}$, and $72{ }^{\circ} \mathrm{C}$ for $28 \mathrm{~s}$. All the reactions were done in triplicate. The relative $\mathrm{T} / \mathrm{S}$ ratio was calculated by the comparative $\mathrm{Ct}$ $\left(2^{-\Delta \Delta_{\mathrm{Ct}}}\right)$ method. To determine the PCR efficiency, a series of twofold dilutions of the reference DNA was added to every plate, and a standard curve was generated. According to the slopes of the standard curves, PCR amplification efficiencies for the target and reference samples were very similar, ranging from 92 to $106 \%$.

\subsection{Measurement of myeloperoxidase}

As previously described [6], a commercial ELISA tests were purchased for the assessment of MPO (EIAab Science Co. Ltd) levels in serum. ELISA tests were performed according to the manufacturer's protocol.

\subsection{Western blot analysis}

PBMCs were lysed in lyses buffer (RIPA) supplemented with complete protease inhibitor cocktail and phosphatase inhibitors (Sigma-Aldrich), and their protein content was determined using the BCA assay (Sigma-Aldrich). For immunoblotting analysis, aliquots of cell extract (15-20 $\mu \mathrm{g}$ ) were electrophoresed on an SDSPAGE and transferred onto a polyvinylidene fluoride membrane
(PVDF, Amersham Biosciences, Milan, Italy). Membranes were blocked with 5\% non-fat dry milk and exposed to the following primary antibodies: Ku80 (\#2180, Cell Signaling) 1:1,000, Bcl-2 (\#2870, Cell Signaling) 1:1,000, Caspase-3 (\#9662, Cell Signaling) $1: 1,000$, TRF2 (sc-9143; Santa Cruz) $1: 1,000, \beta$-actin $(1: 3,000$; Sigma-Aldrich), SIRT1 1:500 (sc-15404, Santa Cruz) 1:1,000, SIRT2 1:500 (sc-20966, Santa Cruz); Bax 1:500 (sc-526, Santa Cruz); TrxR1 1:2,000 (sc-20147, Santa Cruz) and MnSOD 1:1,000 (SOD110, Stressgen). All immunoblots were visualized with horseradish peroxidase-conjugated secondary antibody, followed by detection with enhanced chemiluminescence (Amersham Biosciences). Bands were quantified by Image J software (National Institutes of Health, Bethesda, MD, http://rsb.info.nih.gov/ij). The expression of $\beta$-actin was used as a normalizing control. Detection of primary antibodies was performed using either a mouse or rabbit peroxidase-conjugated HRP (Millipore), and protein signals were visualized using Western Blotting Luminol Reagent (no. 2048; Santa Cruz) and an ImageQuant LAS 4000 (GE Healthcare Life Sciences).

\subsection{Global DNA methylation analysis}

Global DNA methylation analyses were performed using the Imprint Methylated DNA Quantification Kit MDQ1 (Sigma-Aldrich) based on the ELISA principle. We used the 96-well plate format. DNA concentration was diluted to $150 \mathrm{ng} / \mu \mathrm{l}$ in the binding solution. DNA binding was achieved by incubating $30 \mu \mathrm{l}$ diluted DNA (50-100 ng) at $37^{\circ} \mathrm{C}$ for $1 \mathrm{~h}$. A $150 \mu$ l block solution was added, and the samples were incubated at $37^{\circ} \mathrm{C}$ for $30 \mathrm{~min}$. Next, the DNA and block solutions were removed from all the wells, which were washed three times with $150 \mu \mathrm{l}$ of $1 \times$ wash buffers. A $50 \mu \mathrm{l}$ sample of diluted capture antibody was placed in each well and incubated at room temperature for $1 \mathrm{~h}$. After removing the capture antibody and washing four times with the wash buffer, each well was filled with $50 \mu \mathrm{l}$ of diluted detection antibody. The plates were incubated at room temperature for $30 \mathrm{~min}$. The detection antibody was removed from the wells, which were then washed five times with the wash buffer. Each well was then filled with $100 \mu \mathrm{l}$ of developing solution and incubated at room temperature for about 10 min for color change, and subsequently $50 \mu$ l of stop solution was added. The absorbance of each sample was measured at $450 \mathrm{~nm}$. All samples were analysed in triplicate.

\subsection{Statistical analysis}

All statistical analyses were performed using GraphPad Prism 5.0a (GraphPad Software, Inc.) and StatPlus 5.8.2.0 (AnalystSoft, Inc.) program setting a two-tailed $p$ value $\leq 0.05$ as the level of significance.

A prior power analysis was performed by $\mathrm{G}^{*}$ Power 3.1 software [22]. For our experimental design, we used an "effect size f" of 0.19 with an alpha cut-off of 5\% (0.05) and a beta cut-off of 20\% (0.2).

For all parameters analysed, Shapiro-Wilk test was used to check for normal distribution. Repeated measures ANOVA was performed for most of the variables analysed, followed by Bonferroni corrections. Differently, for those variables where the normal distribution was violated (i.e. Bcl-2, MnSOD and MPO) the differences between groups were determined by Friedman test followed Dunns test for post hoc analysis.

The variables measured are represented by box plot or histograms, where every column represents the mean \pm standard error mean (SEM).

Multiple correlation analysis with the Spearman's test was used to evaluate the relationship between "delta variations" of each variable (i.e. LTL, anti-oxidants, oxidative stress parameters, functional tests, power tests and strength test). For all parameters analysed the delta variation was calculated in each subject trough 
the standard formula: $\Delta_{\text {POST-PRE }}=$ POST score - PRE score.

\section{Results}

\subsection{Telomere homeostasis in PBMCs and EMRT}

There were no differences in baseline LTL between CTRL and TR groups (T/S ratio, CTRL $L_{P R E}$ vs. TR $\mathrm{TRE}_{\mathrm{PRE}} 4.9 \pm 0.3$ vs. $4.7 \pm 0.4$, $\mathrm{p}>0.05$ ), without significant differences in the mean $\mathrm{T} / \mathrm{S}$ values between male and female subjects (data not shown). On the contrary, after exercise intervention, the LTL value in TR group resulted statistically higher compared with the corresponding measured in the CTRL group (T/S ratio, CTRL POST Vs. TR $_{\text {POST: POST, }}$ $5.8 \pm 0.3$ vs. $3.9 \pm 0.2, \mathrm{p}<0.05)$. In fact, although no statistical differences in LTL were detected within both experimental groups, at the end of 12-week training (POST) the repeated measures ANOVA revealed a significant different in TL shortening in untrained people with respect the trained ones (Fig. 1).

\subsection{Analysis of Shelterin protein TRF2 and Ku80 following EMRT}

No significant differences were found within and between groups about the effect of EMRT on the protein levels of TRF2 (CTRL ${ }_{\mathrm{PRE}}: 0.73 \pm 0.14 ; \mathrm{CTRL}_{\mathrm{POST}}: 0.62 \pm 0.07 ; \mathrm{TR}_{\mathrm{PRE}}: 0.85 \pm 0.15$; TR $_{\text {POST }}$ : $0.60 \pm 0.11 ; \mathrm{p}>0.05$ ) (Fig. $2 \mathrm{~A}$ ) and Ku80 (CTRL $1.65 \pm 0.50 ; \quad$ CTRL $0.94 \pm 0.35 ; \mathrm{p}>0.05)$ (Fig. 2B).

\subsection{Anti-oxidant proteins expression in circulating PBMCs and ser- um MPO}

As for the analysis of oxidative stress biomarkers in this subsample, we confirmed the results presented in our previous work [22] with respect TrxR1, but also extended the study to MnSOD. Specifically, after the exercise intervention, trained subjects show significantly lower protein expression in PBMCs of MnSOD (CTRL $_{\text {POST Vs. TR }}$ POST: $1.06 \pm 0.06$ vs. $0.82 \pm 0.03, \mathrm{p}<0.01$; TR PRE $_{\text {P v. }}$. TR $_{\text {POST }}: 1.00 \pm 0.04$ vs. $0.82 \pm 0.03, \mathrm{p}<0.01$ ) and TrxR1 (CTRL vs. TR $_{\text {POST }}: 0.85 \pm 0.05$ vs. $0.57 \pm 0.03, \mathrm{p}<0.01$; $\mathrm{TR}_{\mathrm{PRE}}$ : vs. $\mathrm{TR}_{\mathrm{POST}}$ :

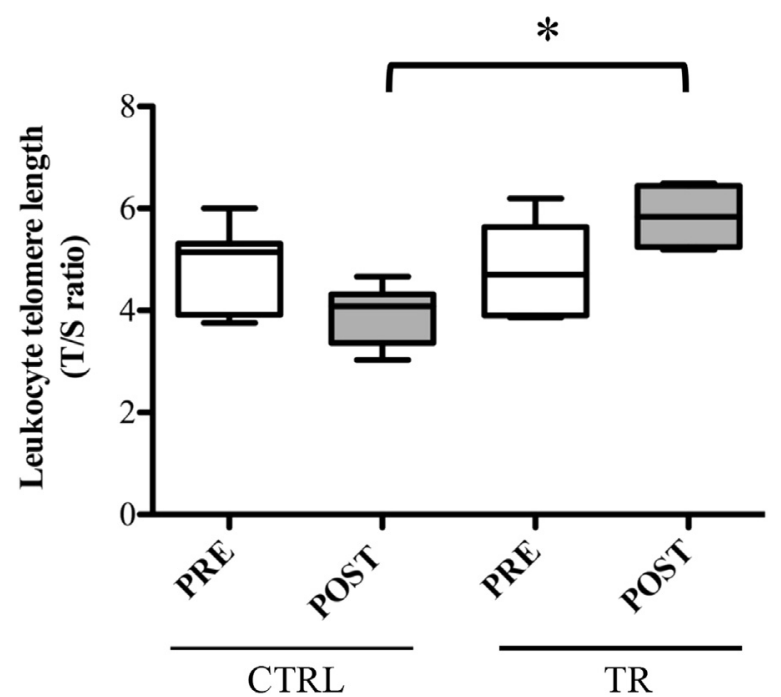

Fig. 1. Analysis of telomere length in PBMCs of elderly people trained for 12 weeks (TR) and those belonging to control group (CTRL). Telomere length was measured at the baseline (PRE) and at the end (POST) of the training protocol in both groups. Boxplots represent the leukocyte telomere length, which is presented in arbitrary units as $\mathrm{T} / \mathrm{S}$ ratio, telomere repeat copy number to single gene copy number. ${ }^{*} \mathrm{p}<0.05$.
$0.79 \pm 0.05$ vs. $0.57 \pm 0.03, \mathrm{p}<0.01$ ) (Fig. $3 \mathrm{~A}-\mathrm{B}$ ), paralleled by a decreased level of MPO in serum (CTRL vs. $76.8 \pm 4.1, \mathrm{p}<0.05$; $\mathrm{TR}_{\mathrm{PRE}}$ : vs. $\mathrm{TR}_{\mathrm{POST}}$ : $89.1 \pm 6.3$ vs. $76.8 \pm 4.1$, $\mathrm{p}<0.05$ ) (Fig. 3C).

\subsection{Effect of EMRT on global DNA methylation}

Comparative analysis of the global DNA methylation level revealed no significant differences within the CTRL group (CTRL $\mathrm{PRE}_{\mathrm{PE}}$ : $0.44 \pm 0.07$ vs. CTRL ${ }_{\text {POST }}: 0.45 \pm 0.08, \mathrm{p}>0.05$ ) or with TR group at

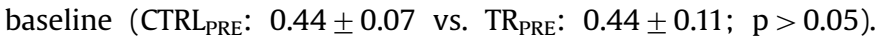
Differently, the global DNA methylation level was significantly decreased after EMRT within the TR group (TR $\mathrm{PRE}_{\mathrm{PR}}$ : $0.44 \pm 0.11 \mathrm{vs}$. $\left.\mathrm{TR}_{\mathrm{POST}}: 0.36 \pm 0.07,-18 \% \mathrm{p}<0.05\right)$ and compared with the corresponding experimental time point in the CTRL group (CTRLPOST

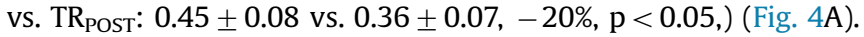

3.5. Effect of EMRT on SIRT1 and SIRT2 protein expression and total protein acetylation in PBMCs

Although SIRT1 protein expression demonstrated a trend toward an increase following exercise within the TR group ( $\mathrm{TR}_{\mathrm{PRE}}$ : $0.79 \pm 0.13$ vs. TR $_{\text {POST }}: 1.25 \pm 0.13, \mathrm{p}=0.059$ ), the statistical analysis has failed to find significant differences within and between groups, at any experimental time points (CTRL $\mathrm{PRE}_{\mathrm{PE}}$ : $0.78 \pm 0.19$; CTRL POST $: 0.89 \pm 0.17, \mathrm{p}>0.05$ ) (Fig. $4 \mathrm{~B}$ ).

Similarly, SIRT2 expression did not reach any significant change after EMRT (CTRL ${ }_{\text {PRE: }} 0.97 \pm 0.12$; TR PRE $_{1}$ : $1.22 \pm 0.10$; CTRLPOST: $1.11 \pm 0.12$; TR $\left._{\text {POST }}: 1.13 \pm 0.13\right)(\mathrm{p}>0.05)$ (Fig. $\left.4 \mathrm{C}\right)$.

Still, for both experimental groups, any significant change has been found for the total protein acetylation either before or after exercise intervention (CTRL ${ }_{\mathrm{PRE}}: 8.71 \pm 2.38$; $\mathrm{TR}_{\mathrm{PRE}}$ : $5.16 \pm 1.40$; CTRL ${ }_{\text {POST }}: 7.46 \pm 2.40$; TR

\subsection{Expression of pro- and anti-apoptotic markers in PBMCs fol-} lowing EMRT

With respect the pro- and anti-apoptotic biomarkers analysed, no significant changes were found within and between CTRL and TR groups at any experimental time points: Bcl-2 (CTRLPRE: $1.02 \pm 0.22 ; \mathrm{TR}_{\mathrm{PRE}}: 0.75 \pm 0.23 ; \mathrm{CTRL}_{\mathrm{POST}}: 0.65 \pm 0.27 ; \mathrm{TR}_{\mathrm{POST}}$ : $0.71 \pm 0.24) \quad(\mathrm{p}>0.05) ; \quad$ Bax $\quad\left(\mathrm{CTRL}_{\mathrm{PRE}}: \quad 0.71 \pm 0.08 ; \quad \mathrm{TR}_{\mathrm{PRE}}:\right.$

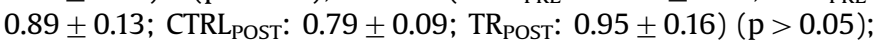

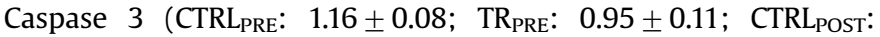
$0.91 \pm 0.10$; TR POST: $_{1.03 \pm 0.09)}(\mathrm{p}>0.05)$ (Fig. $\left.5 \mathrm{~A}-\mathrm{C}\right)$.

\subsection{Correlation between telomere length with molecular and func-} tional parameters

Table 2 shows the results from the correlation analysis between LTL and all the molecular or functional parameters analysed in this protocol at individual level. A significant negative correlation was found between LTL and TrxR1 expression in PBMC $(r=-0.615$, $\mathrm{p}=0.006)$ or serum MPO levels $(r=-0.754, \mathrm{p}=0.00092)$, while no correlation emerged with MnSOD, as well as with all the other molecular markers. When taking in consideration functional parameters, a significant positive correlation was observed between LTL with functional power test (CMJ: $r=0.786, \mathrm{p}=0.0003$ ) and strength test (LegExt: $r=0.655, \mathrm{p}=0.004$ ), while negative correlation was detected with $6 \mathrm{~m}$ walking speed $(r=-0.516$, $\mathrm{p}=0.029), 6 \mathrm{~m}$ walking loaded $(r=-0.468, \mathrm{p}=0.046)$ and loaded stairs climbing $(r=-0.521, \mathrm{p}=0.028)$.

\section{Discussion}

The exact influence of regular resistance training on DNA 
A

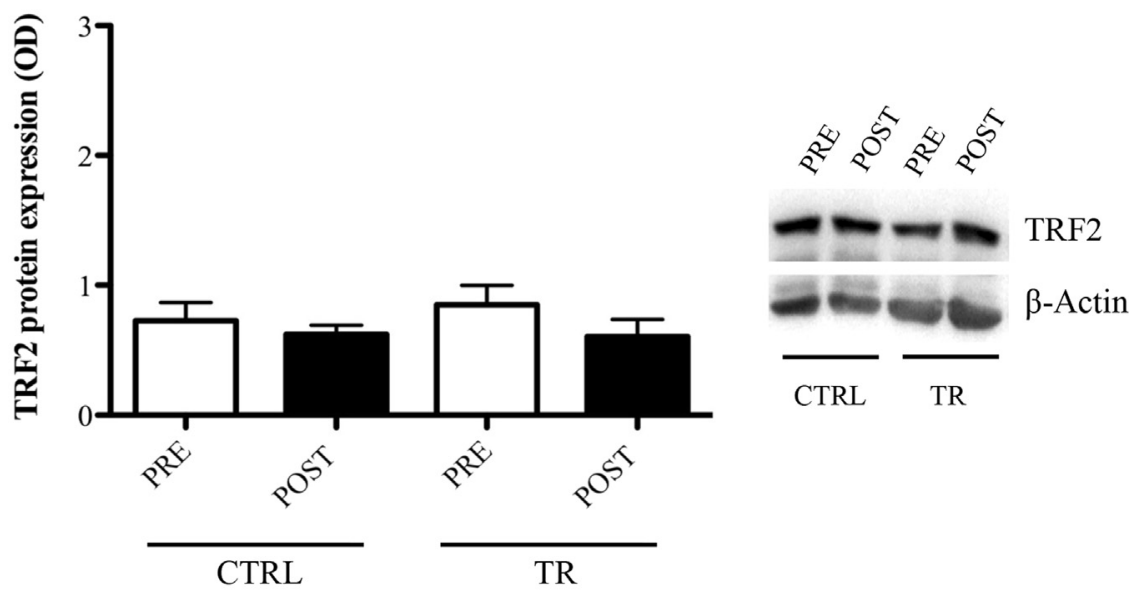

B

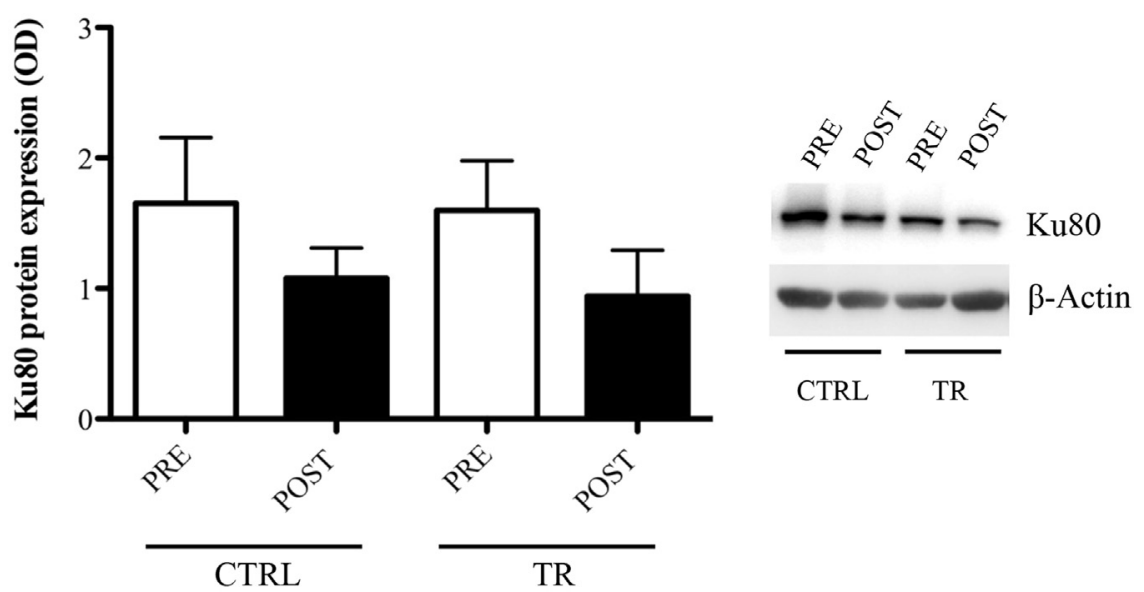

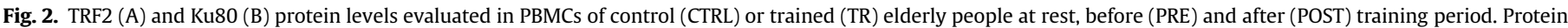

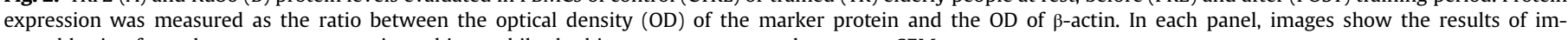
munoblotting from the same representative subject, while the histograms represent the mean \pm SEM.

telomere ends in PBMCs collected from elderly subjects has never been addressed. Here, we present a longitudinal study where we observed that: a) regular participation to EMRT of healthy elderly people is associated with a better maintenance of LTL and reduction of the global DNA methylation level; b) EMRT induces an adaptation of specific anti-oxidants/oxidative stress markers; c) LTL is significantly correlated to markers of redox homeostasis as well as to several indicators of physical functioning.

Exercise can influence aging through its preventive effects on various diseases [70]. It has been suggested that numerous beneficial effects of exercise may be related to $\operatorname{LTL}[14,37,18]$ and to the modulation of the epigenome, which thereby may induce the adaptation of several stress-proteins/molecules response (i.e. antioxidants, heat shock proteins) already observed in different studies $[16,47,6,7,9]$.

Telomere shortening and age-dependent alteration of specific epigenetic pattern are widely considered to be a marker of health status and biological ageing because they are recognized as important factors able to affect the risk for several human diseases $[19,24,36]$. Indeed, both processes may be considered a dynamic feature, which can undergo to considerable changes over several months in response to environmental, behavioral, psychological and pathological stimuli [24], as well as during a rather short period of time of physical activity [66].

PBMCs are a proliferative cell type in which replication leads to continued decrease of telomere length, and thus correlates well with aging [31]. To date, it is still difficult to compare the relative leukocyte telomere length data because of differences in qPCR methodology as well as the different subject population and sample sizes. Moreover, other discrepant findings are partly due to differences in physical activity measurements for intensity and duration of exercise, and/or because a self-reported approach has been utilized. Although Mason and colleagues have found discordant results, several studies have shown that LTL is associated with the level of PA and maximal aerobic exercise capacity $[14,17,42]$. Indeed, all these studies agree on the fact that endurance training could ameliorate the telomere length homeostasis in a PA-intensity and frequency dependent manner.

The ageing process is characterized by a reduction in motor capacity due to a gradual decrease of muscle strength and power [23]. It is known that resistance training can counteract physiological and biological age-related negative changes improving the performance of functional capacities and the stress oxidative/ protein response $[45,51,6]$, but the proportion of elderly people who participate in this type of training protocol is currently low.

Here, we present an interventional study evaluating the effect of resistance-type exercise on LTL in healthy elderly people. Similarly to the aforementioned studies [14,17,39], we found that participation to low frequency, moderate intensity power training affects the telomeres in old age, resulting in a reduction of 
A
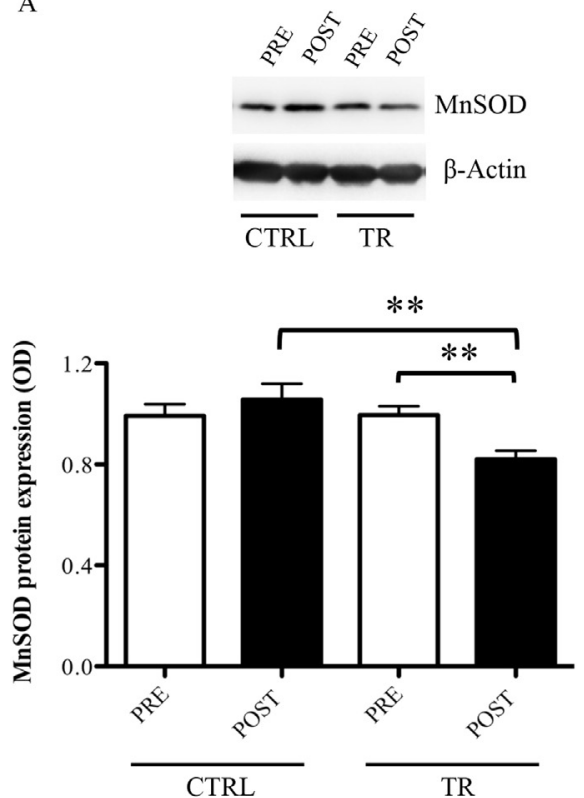

B
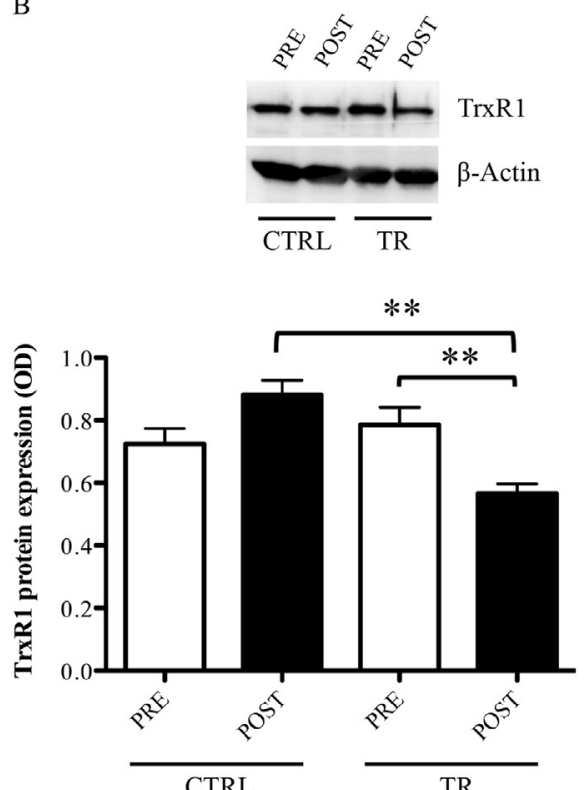

CTRL
$\mathrm{C}$

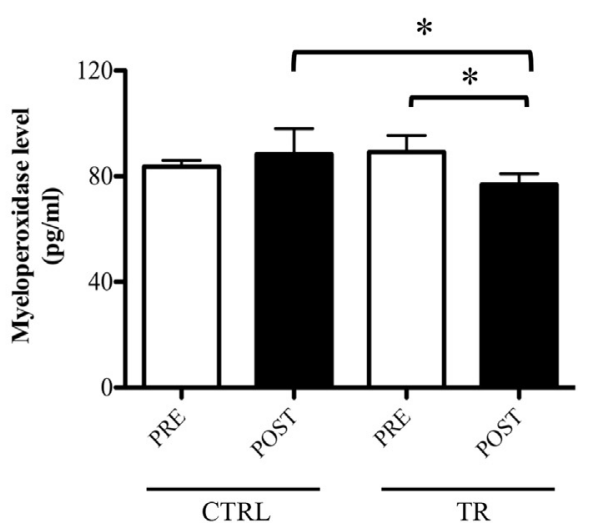

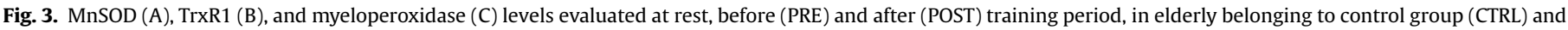

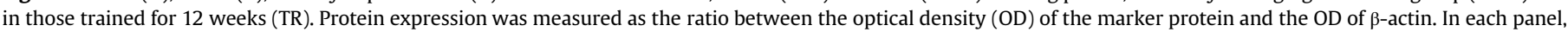
images show the results of immunoblotting from the same representative subject, while the histograms represent the mean \pm SEM. ${ }^{* *} \mathrm{p}<0.01$ and ${ }^{*} \mathrm{p}<0.05$.

telomere's attrition when compared to age-matched subjects that have not participated in any exercise training. There are several mechanisms that might explain TL maintenance in exercised subjects. For instance, the telomerase enzyme and other capping proteins called the shelterin complex are involved in telomere maintenance (i.e. TRF1, TRF2, Ku70, Ku80) [48]. Since previous research protocols did not found any relationship between PA level and telomerase enzyme activity in PBMCs [37,49], we decided to exclude the analysis of telomerase activity. Indeed, in certain cells with high mitotic activity (e.g., germ line and progenitor cells, immune cells), the elevated activity of telomerase enzyme due for the telomere length's homeostasis could blunt the effect of PA. Although the relationship between telomerase enzyme activity and PA deserves deep investigations, we considered more appropriate to investigate whether EMRT alters the protein expression of members of the shelterin complex, such as the telomere-related factors 2 (TRF2) and Ku80. Similarly to previous studies [38], any alteration of these proteins was observed in PBMCs. Since it has been described that the mRNA level of these markers is induced in circulating leukocyte either after a seven day ultra-marathon [46] or in athletes [40], we speculate that the absence of TRF2 and/or Ku80 protein induction could be due either to the lower intensity of our exercise protocol or/and the characteristic of the subjects. However, we cannot exclude the possibility that a similar protocol with longer duration than 3 months could modulate the protein expression of these members of shelterin complex.

Another important factor that enhances age-related telomere shortening is the oxidative stress level [56], a common feature of the ageing process with a strong correlation to several pathological conditions (i.e. diabetes, metabolic disorders) [29]. Several studies showed that PA could reduce oxidative stress $[16,3,47,59]$. In particular, moderate-intensity of PA over a 2- or 12-month period has shown to be effective to reduce gene and protein expression of several "actors" of the anti-oxidant response in PBMCs $[6,9]$. In agreement with aforementioned studies, we found that the levels of specific proteins belonging to anti-oxidant/oxidative stress system, such as the member of iron/manganese superoxide dismutase family, MnSOD [74], the cellular redox sensor TrxR1 [64], and the clinical biomarker of oxidative stress MPO [58], were significantly reduced in trained subjects.

It is known that exercise-induced adaptation of antioxidant is dependent from several variables, which include tissue, age, duration, intensity and exercise mode specific [55]. Data on the effect of exercise training on the behavior of MnSOD enzyme in people matching for the age with our participants, are limited to specific experimental conditions, which make the comparison between them difficult and potentially confounding [2,12,34,44]. In particular, most human studies examine MnSOD modulation in skeletal muscle (i.e. protein expression), utilizing endurance exercise as interventional training program [12,34]. Moreover, the MnSOD response to exercise appears to be heterogeneous.

To our knowledge, the only study documenting the effect of resistance training on MnSOD in peripheral blood mononuclear cells (PBMC), has been conducted by García-López et al. [26] but the subjects recruited were younger (age $54.9 \pm 1.9$ ) than those included in our research protocol.

Nevertheless, based on their findings, it has been hypothesized that resistance training generally results in a cytosolic oxidative stress [26]. Since MnSOD is compartmentalized in the mitochondrial inner membrane, its lack induction or decreased expression after resistance training may simply represent an adaptive mechanism related to the fact that this type of exercise is not associated with mitochondrial stress. However, we cannot exclude that the cytosolic anti-oxidant enzyme may be more responsive to exercise and/or greater exercise intensity is needed to elicit an induction of MnSOD.

Therefore, it is reasonable to assume that, perhaps facilitated by a better homeostasis redox, decreased levels of MnSOD and TrxR1, represent an adaptive response to our exercise training. As already suggested by von Zglinicki [68], exercise might buffer telomere shortening by acting on the balance between oxidative stress and antioxidant efficiency. Our results confirm that EMRT was paralleled by an improvement of the redox homeostasis, in turn leading to an adaptive process induced by exercise that may influence positively the telomere length in leukocytes. Indeed, our data indicate a significant, negative correlation between TrxR1 or MPO and LTL. The biological mechanisms of these correlations are not clear, but considering the context of this study, our results may be 


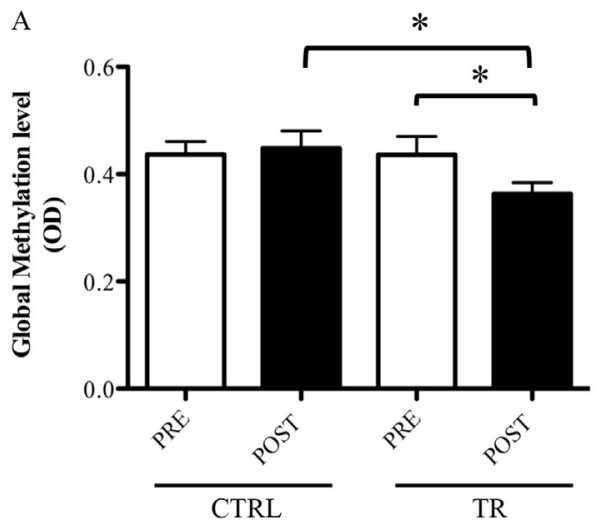

B
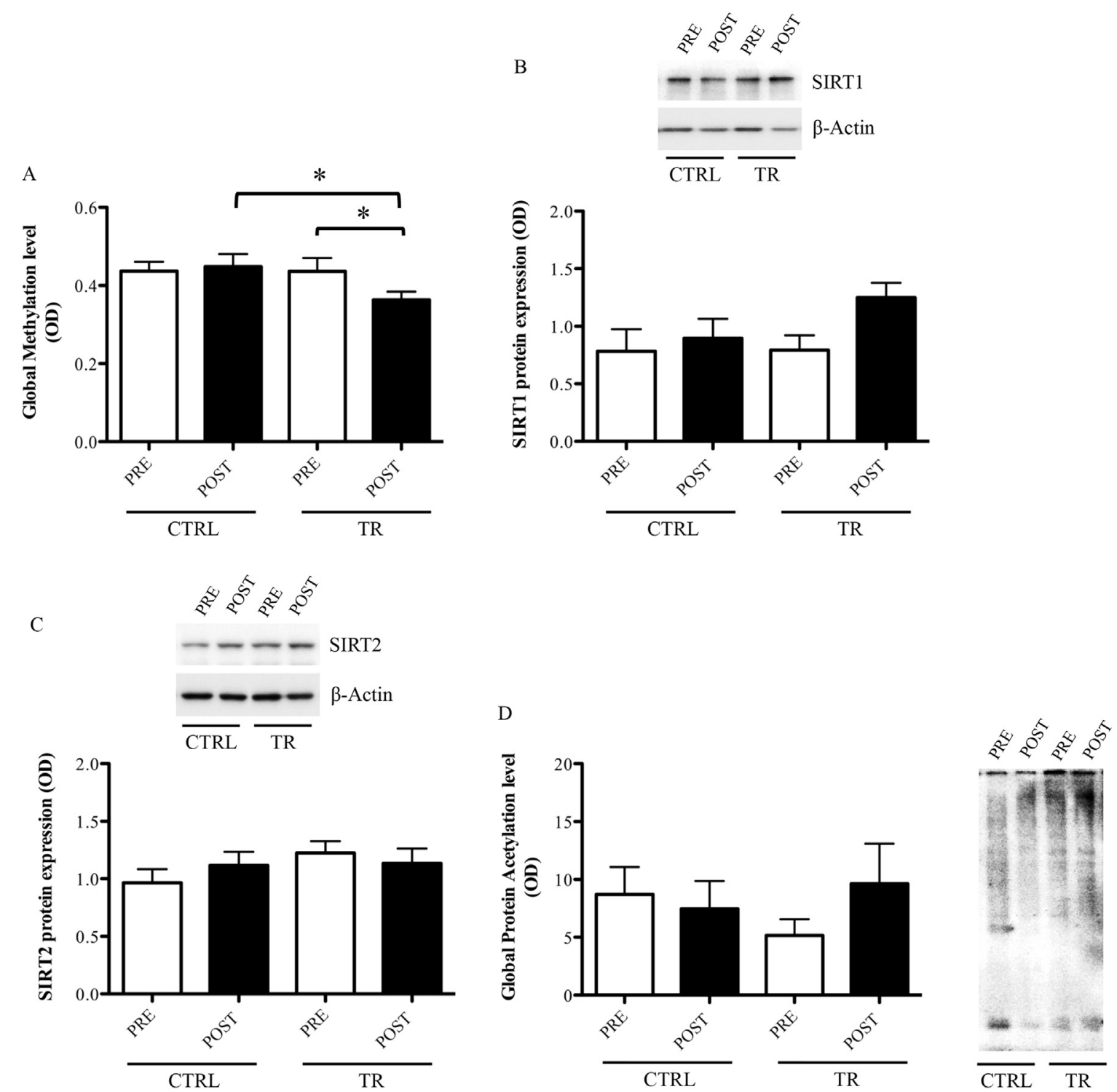

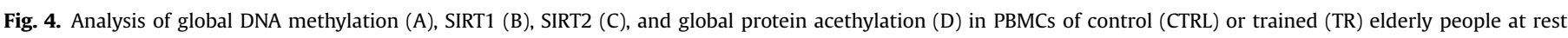

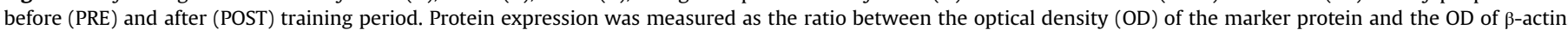
Each histogram represents the mean \pm SEM. ${ }^{*} \mathrm{p}<0.05$.

explained by the exercise-induced adaptation toward an optimal redox homeostasis, thereby enabling a lower erosion of susceptible telomere ends $[68,63]$.

Contradictory findings exist with respect the association between LTL and specific indicators of physical functioning $[27,28,73,61]$, primarily in elderly population. However, most of the data support the hypothesis that this sensitive chromosome region may be considered a biomarker of age-related changes in functional capacity. In agreement with these studies [27,28,73,61], we found not only a significant correlation between LTL and the functional capacities such as $6 \mathrm{~m}$ walk speed with or without load, stairs climbing with load, but also was highlighted an association with the muscle power (CMJ) and muscle strength capacities (LegExt). Although speculative, it is possible that the sustained weight-bearing aspect of EMRT protocol may be optimal in activating specific signaling pathways involved in telomere homeostasis.

It is also known that the immune system response to exercise is transient and variable because is influenced by a range of factors such as duration, intensity and mode of the exercise [60]. Since previous studies have shown an increased apoptosis of immune system cells in aging [1], and that exercise can have anti-apoptotic effect that reduces cellular turnover and maintains telomere length [40], we investigated if EMRT could have an impact on the commitment of apoptosis in PBMCs. In agreement with Rodriguez Silva group [62], our exercise training did not affect the survival of circulating mononuclear cells. Indeed, the expression of antiapoptotic (Bcl-2) and pro-apoptotic (Bax, Caspase-3) proteins showed considerable intragroup variability and no significant differences between groups. Although we cannot rule out the contribution of new lymphocyte populations in maintaining the telomere ends longer, these data supports even more that this exercise program can contribute to a better preservation of the telomere ends. Further studies are needed to understand the precise immunological mechanisms connecting TL and immune response. However, to date preserve the telomere length has also a positive impact on the quality of the immune response [15].

The physiological adaptation to exercise is predominantly driven by changes in gene expression, which may be governed by exercise-induced changes to the epigenome [75]. It is known that epigenetic factors, including DNA methylation and histone modifications, are influenced by relative short exposure to 
A

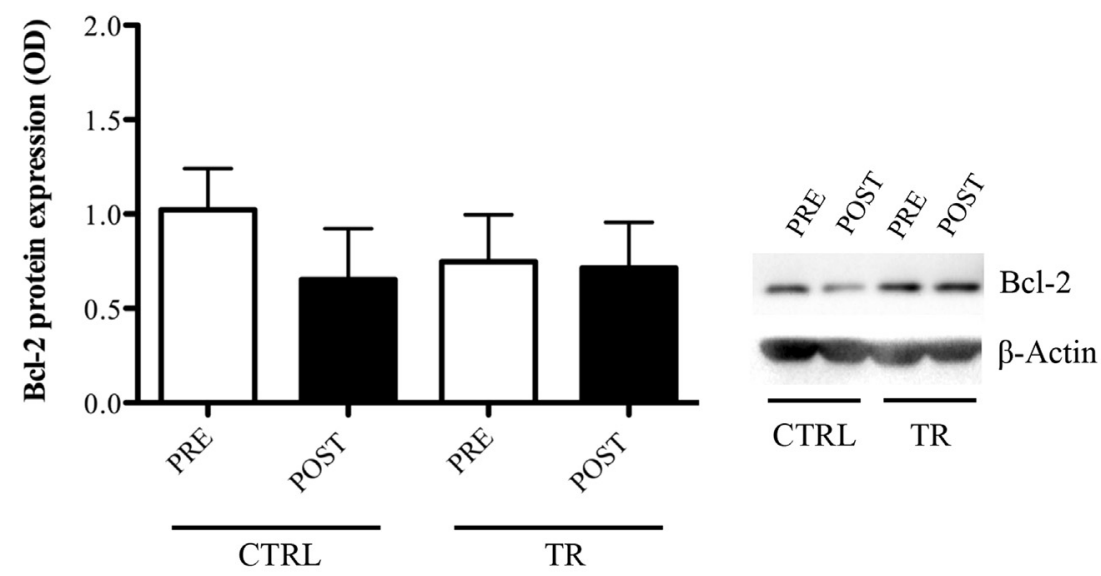

B
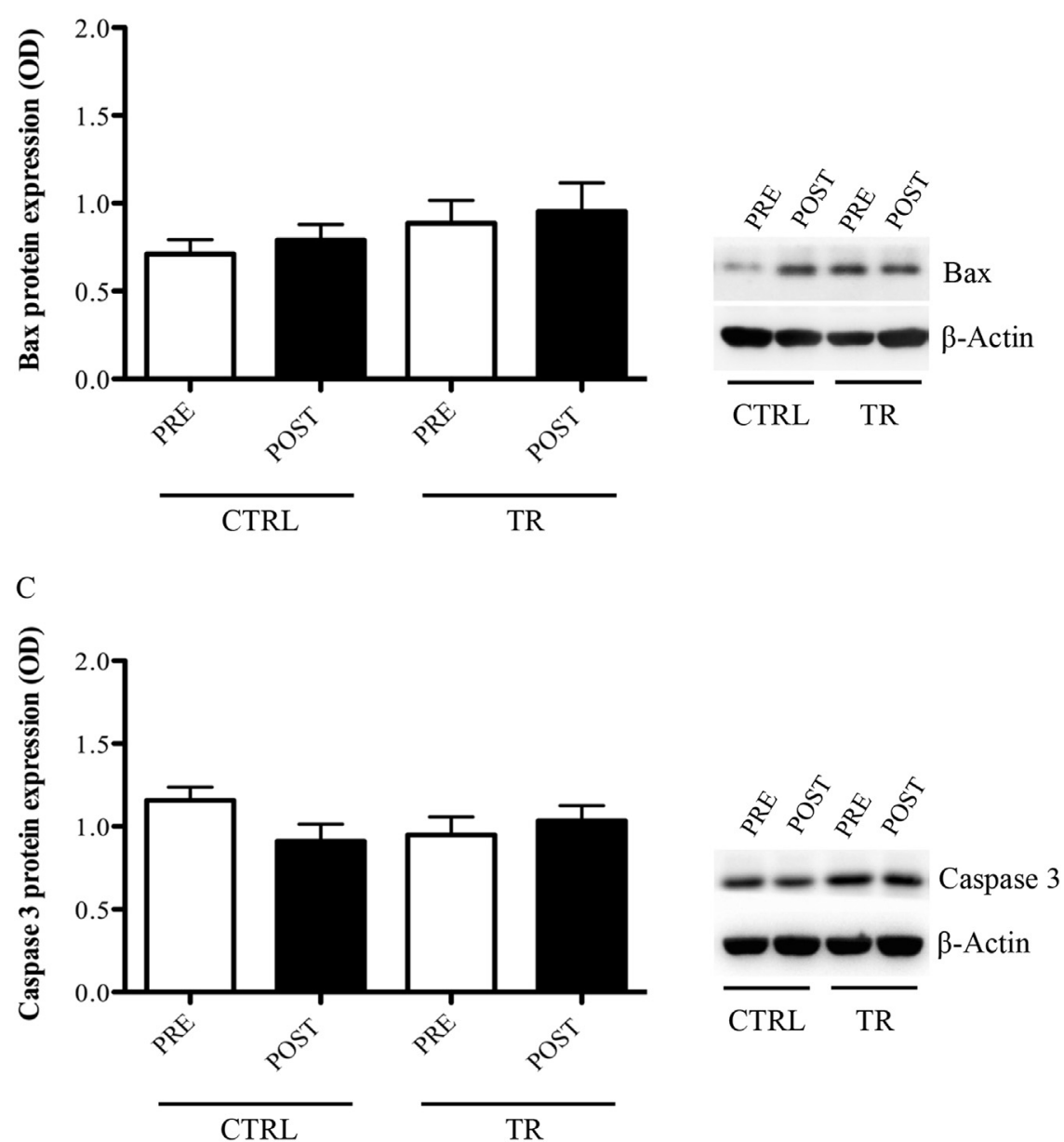

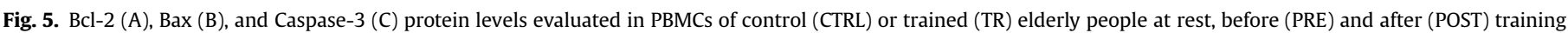

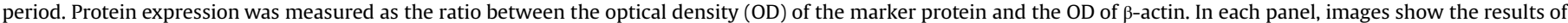
immunoblotting from the same representative subject, while the histograms represent the mean \pm SEM.

environmental stimuli such as exercise [32]. Buxton and colleagues [8] have also observed a correlation between telomere length and DNA methylation of sub-telomeric regions, indicating that as telomeres shorten, the methylation levels of many genes may change, inducing modification in gene expression with an increased age-related disease risk. In order to evaluate the potential of our physical exercise program in modulating stress-induced epigenetic changes, we have investigated for the first time its effect on global DNA methylation status, SIRT1, and SIRT2 protein expression as well as the global protein acetylation level in PBMCs.

Although the site-specific changes in DNA methylation of $\mathrm{CpG}$ would give a more detailed and complete information about the genes potentially involved, our data revealed that EMRT evoked a decrease in the global DNA methylation profile in exercised people. Exercise, either a single session or a training period, has been shown to affect DNA methylation status in several tissues (i.e. 
Table 2

Linear correlation between leukocyte telomere length and biological/functional parameters.

\begin{tabular}{lll}
\hline & $\boldsymbol{r}$ & $\boldsymbol{P}$ \\
\hline Biological parameters & & \\
TrxR1 & $-\mathbf{0 . 6 1 5}$ & $\mathbf{0 . 0 0 6}$ \\
MnSOD & -0.291 & 0.167 \\
MPO & $-\mathbf{0 . 7 5 4}$ & $\mathbf{0 . 0 0 1}$ \\
SIRT1 & 0.202 & 0.292 \\
SIRT2 & 0 & 0.5 \\
TRF2 & -0.136 & 0.347 \\
Ku80 & 0.394 & 0.132 \\
Global protein acetylation & 0.452 & 0.134 \\
Caspase-3 & 0.297 & 0.203 \\
Bcl-2 & 0.357 & 0.195 \\
Bax & 0.417 & 0.135 \\
Functional parameters & & \\
CMJ & $\mathbf{0 . 7 8 6}$ & $\mathbf{0 . 0 0 0 3}$ \\
LegExt & $\mathbf{0 . 6 5 5}$ & $\mathbf{0 . 0 0 4}$ \\
6 m walking speed & $-\mathbf{0 . 5 1 6}$ & $\mathbf{0 . 0 2 9}$ \\
6 m walking loaded & $-\mathbf{0 . 4 6 8}$ & $\mathbf{0 . 0 4 6}$ \\
Stairs climbing & -0.235 & 0.209 \\
Stairs climbing loaded & $-\mathbf{0 . 5 2 1}$ & $\mathbf{0 . 0 2 8}$ \\
\hline
\end{tabular}

Data from Spearman's correlations are expressed by $r$ and $p$-values.

MPO, Myeloperoxidase; TrxR1, Thioredoxin reductase 1; MnSOD, Manganese-dependent superoxide dismutase; SIRT1, Sirtuin 1; SIRT2, Sirtuin 2; TRF2, Telomeric repeat-binding factor 2; CMJ, countermovement jump; LegExt, Leg extension.

${ }^{*} \mathrm{P}<0.05$ was considered significant.

skeletal muscle, PBMCs, adipose tissue) that results in gene expression changes $[4,52,53]$. Indeed, hypomethylation of a gene's promoter $\mathrm{CpG}$ region is associated with transcriptional activation of genes capable of improving the process of adaptation to the new physiological or pathological condition [2].

Therefore, our finding suggests that the resistance exercise intervention impacts significantly on global methylation levels, and thereby altering the gene activity. Since recent finding has identified multiple gene promoters enriched for site at which methylation levels are associated with TL [4], it remains definitely needed a more detailed study to identify individual CpG-sites potentially associated either with common diseases or with telomere length homeostasis in PBMCs following EMRT.

Much attention has recently been given to the role of sirtuins as regulator of chromatin remodeling, gene silencing, and DNA damage response. Recent findings have shown that not only the increased expression of SIRT1 in mice results in longer telomeres and a major telomere integrity following DNA damage [33], but also in human subjects Kim and colleagues [35] showed an association between SIRT1 and telomere length. Increasing evidences has indicated that sirtuins are regulated by exercise in human and animal model [65]. However, to date, only one human study has demonstrated an induction of SIRT1 mRNA level in PBMCs [41]. Despite we found a tendency to increase of SIRT1 protein level in elderly after the training, no significant changes were obtained for both sirtuins and global protein acetylation.

Since there are several mechanisms by which aerobic exercise may regulate sirtuins expression, such as the production of nitric oxide (NO) and NO synthases or the increase of energy expenditure [54], differently from previous studies [41,65], EMRT characteristics probably were not enough specific to modify these parameters. Nevertheless, we cannot exclude that a resistance exercise protocol prolonged over time could have similar effects to those observed in endurance exercises.

\section{Study limitations and conclusions}

Our study findings should be interpreted bearing in mind some limitations. Firstly, with regard to telomere length and EMRT, we cannot exclude the possibility of residual confounding because of unknown or unmeasured factors in the present study such as information on psychological stress [20] as well as antioxidant intake and genetic variants [13].

Secondly, in some instances, the small sample size may have reduced, the number of statistically significant differences (e.g. SIRT1). Still, it would be interesting to analyze age-related markers even during the training period because without a priori knowledge of the precise time course of protein expression, it is possible that the greatest magnitude of change has been missed.

In conclusion, our present results corroborate the evidence that resistance training seems to have beneficial systemic 'anti-aging' effects even in term of DNA methylation modification and telomere dynamics, while a life style with no or irregular PA is linked with an increasing decline of the molecular parameters related to the aging process. To date, many of these biomarkers are known to describe more accurately the development of the biological aging process rather that chronological age [43], and therefore, they may serve to identify subjects at risk for accelerated age-related adverse outcomes. For these reasons, further investigation on the effects of specific exercise protocol on these markers as well as the delineation of molecular pathways responsible for their modifications (e.g. TL erosion and site-specific DNA methylation), are doubtless of high priority.

\section{Conflict of interest statement}

The authors declare that there are not conflicts of interest.

\section{Funding}

This work was supported by grants from MIUR (PRIN2012) to DC and from University of Rome "Foro Italico" (Research Grant 2013) to LDL. Further, we thank the "Veronesi Foundation" for the fellowships given to Dr. Ivan Dimauro

\section{Acknowledgments}

We thank Dr. Paola Teti from University of Rome "Foro Italico", for the English spelling revision.

\section{References}

[1] S. Aggarwal, S. Gupta, Increased apoptosis of T cell subsets in aging humans: altered expression of Fas (CD95), Fas ligand, Bcl-2, and Bax, J. Immunol. 160 (1998) 1627-1637.

[2] O.S. Anderson, K.E. Sant, D.C. Dolinoy, Nutrition and epigenetics: an interplay of dietary methyl donors, one-carbon metabolism and DNA methylation, J. Nutr. Biochem. 23 (2012) 853-859.

[3] S. Attipoe, J.Y. Park, N. Fenty, D. Phares, M. Brown, Oxidative stress levels are reduced in postmenopausal women with exercise training regardless of hormone replacement therapy status, J. Women Aging 20 (2008) 31-45.

[4] R. Barrès, J. Yan, B. Egan, et al., Acute exercise remodels promoter methylation in human skeletal muscle, Cell. Metab. 15 (2012) 405-411.

[5] A. Bauman, D. Merom, F.C. Bull, D.M. Buchner, M.A. Fiatarone Singh, Updating the evidence for physical activity: summative reviews of the epidemiological evidence, prevalence, and interventions to promote "Active Aging", Gerontologist 256 (2016) S268-S280.

[6] M.R. Beltran Valls, I. Dimauro, A. Brunelli, et al., Explosive type of moderateresistance training induces functional, cardiovascular, and molecular adaptations in the elderly, Age 36 (2014) 759-772, http://dx.doi.org/10.1007/ s11357-013-9584-1.

[7] A. Brunelli, I. Dimauro, P. Sgrò, et al., Acute exercise modulates BDNF and proBDNF protein content in immune cells, Med. Sci. Sports Exerc. 44 (2012) 1871-1880.

[8] J.L. Buxton, M. Suderman, J.J. Pappas, et al., Human leukocyte telomere length is associated with DNA methylation levels in multiple subtelomeric and 
imprinted loci, Sci. Rep. 14 (2014) 4-4954

[9] P.T. Campbell, M.D. Gross, J.D. Potter, et al., Effect of exercise on oxidative stress: a 12-month randomized, controlled trial, Med. Sci. Sports Exerc. 42 (2010) 1448-1453.

[10] R.M. Cawthon, Telomere measurement by quantitative PCR, Nucl. Acids Res. 30 (2002) e47.

[11] R. Ceci, M.R. Beltran Valls, G. Duranti, et al., Oxidative stress responses to a graded maximal exercise test in older adults following explosive-type resistance training, Redox Biol. 12 (2013) 65-72, http://dx.doi.org/10.1016/j. redox.2013.12.004.

[12] J.N. Cobley, G.K. Sakellariou, D.J. Owens, et al., Lifelong training preserves some redox-regulated adaptive responses after an acute exercise stimulus in aged human skeletal muscle, Free Radic. Biol. Med. 70 (2014) 23-32.

[13] V. Codd, C.P. Nelson, E. Albrecht, et al., Identification of seven loci affecting mean telomere length and their association with disease, Nat. Genet. 45 (2013) 422-427.

[14] L.F. Cherkas, J.L. Hunkin, B.S. Kato, et al., The association between physical activity in leisure time and leukocyte telomere length, Arch. Intern. Med. 168 (2008) 154-158.

[15] S. Cohen, D. Janicki-Deverts, R.B. Turner, et al., Association between telomere length and experimentally induced upper respiratory viral infection in healthy adults, JAMA 309 (2013) 699-705.

[16] P.H. Connolly, V.J. Caiozzo, F. Zaldivar, et al., Effects of exercise on gene expression in human peripheral blood mononuclear cells, J. Appl. Physiol. 97 (2004) 1461-1469.

[17] J. Denham, C.P. Nelson, B.J. O'Brien, et al., Longer leukocyte telomeres are associated with ultra-endurance exercise independent of cardiovascular risk factors, PLoS One 8 (2013) e69377.

[18] M. Du, J. Prescott, P. Kraft, et al., Physical activity, sedentary behavior, and leukocyte telomere length in women, Am. J. Epidemiol. 175 (2012) 414-422.

[19] D.T. Eisenberg, An evolutionary review of human telomere biology: the thrifty telomere hypothesis and notes on potential adaptive paternal effects, Am. J. Hum. Biol. 23 (2011) 149-167.

[20] E.S. Epel, E.H. Blackburn, J. Lin, et al., Accelerated telomere shortening in response to life stress, Proc. Natl. Acad. Sci. USA 101 (2004) 17312-17315.

[21] R. Farzaneh-Far, R.M. Cawthon, B. Na, W.S. Browner, N.B. Schiller, M. A. Whooley, Prognostic value of leukocyte telomere length in patients with stable coronary artery disease: data from the heart and soul study, Arterioscler Thromb. Vasc. Biol. 28 (2008) 1379-1384.

[22] F. Faul, E. Erdfelder, A.G. Lang, A. Buchner, G*Power3: a flexible statistical power analysis program for the social, behavioral, and biomedical sciences, Behav. Res. Methods 39 (2007) 175-191.

[23] J.A. Faulkner, L.M. Larkin, D.R. Claflin, S.V. Brooks, Age-related changes in the structure and function of skeletal muscles, Clin. Exp. Pharmacol. Physiol. 34 (2007) 1091-1096.

[24] A.P. Feinberg, R.A. Irizarry, Evolution in health and medicine Sackler colloquium: stochastic epigenetic variation as a driving force of development, evolutionary adaptation, and disease, Proc. Natl. Acad. Sci. USA 107 (2010) $1757-1764$.

[25] U. Friedrich, E.U. Griese, M. Schwab, et al., Telomere length in different tissues of elderly patients, Mech. Ageing Dev. 119 (2000) 89-99.

[26] D. García-López, K. Häkkinen, M.J. Cuevas, et al., Effects of strength and endurance training on antioxidant enzyme gene expression and activity in middle-aged men, Scand. J. Med. Sci. Sports 17 (2007) 595-604.

[27] M.P. Gardner, C. Martin-Ruiz, R. Cooper, et al., Telomere length and physical performance at older ages: an individual participant meta-analysis, PLoS One 8 (2013) e69526.

[28] S.E. Harris, I.J. Deary, A. MacIntyre, et al., The association between telomere length, physical health, cognitive ageing, and mortality in non-demented older people, Neurosci. Lett. 406 (2006) 260-264.

[29] J.M. Houben, H.J. Moonen, F.J. van Schooten, G.J. Hageman, Telomere length assessment: biomarker of chronic oxidative stress? Free Radic. Biol. Med. 44 (2008) 235-246.

[30] J.P. Issa, Age-related epigenetic changes and the immune system, Clin. Immunol. 109 (1) (2003) 103-108.

[31] H. Iwama, K. Ohyashiki, J.H. Ohyashiki, et al., Telomeric length and telomerase activity vary with age in peripheral blood cells obtained from normal in dividuals, Hum. Genet. 102 (1998) 397-402.

[32] R. Jaenisch, A. Bird, Epigenetic regulation of gene expression: how the genome integrates intrinsic and environmental signals, Nat. Genet. 33 (2003) 245-254.

[33] J. Jeong, K. Juhn, H. Lee, et al., SIRT1 promotes DNA repair activity and deacetylation of Ku70, Exp. Mol. Med. 39 (2007) 8-13.

[34] M.L. Johnson, B.A. Irving, I.R. Lanza, et al., Differential effect of endurance training on mitochondrial protein damage, degradation, and acetylation in the context of aging, J. Gerontol. A Biol. Sci. Med. Sci. 70 (2015) 1386-1393.

[35] S. Kim, X. Bi, M. Czarny-Ratajczak, et al., Telomere maintenance genes SIRT1 and XRCC6 impact age-related decline in telomere length but only SIRT1 is associated with human longevity, Biogerontology 13 (2012) 119-131.

[36] C.M. Kong, X.W. Lee, X. Wang, Telomere shortening in human diseases, FEBS J. 280 (2013) 3180-3193.

[37] T.J. LaRocca, D.R. Seals, G.L. Pierce, Leukocyte telomere length is preserved with aging in endurance exercise-trained adults and related to maximal aerobic capacity, Mech. Ageing Develop. 131 (2010) 165-167.

[38] M.J.1 Laye, T.P. Solomon, K. Karstoft, K.K. Pedersen, S.D. Nielsen, B.K. Pedersen, Increased shelterin mRNA expression in peripheral blood mononuclear cells and skeletal muscle following an ultra-long-distance running event, J. Appl.
Physiol. 2012 (112) (1985) 773-781

[39] P.D. Loprinzi, E. Sng, Mode-specific physical activity and leukocyte telomere length among U.S. adults: implications of running on cellular aging, Prev. Med. 85 (2016) 17-19.

[40] A.T. Ludlow, J.B. Zimmerman, S. Witkowski, J.W. Hearn, B.D. Hatfield, S. M. Roth, Relationship between physical activity level, telomere length, and telomerase activity, Med. Sci. Sports Exerc. 40 (2008) 1764-1771.

[41] G. Marfe, M. Tafani, B. Pucci, et al., The effect of marathon on mRNA expression of anti-apoptotic and pro-apoptotic proteins and sirtuins family in male recreational long-distance runners, BMC Physiol. 10 (2010) 7.

[42] C. Mason, R.A. Risques, L. Xiao, C.R. Duggan, I. Imayama, K.L. Campbell, A. Kong, K.E. Foster-Schubert, C.Y. Wang, C.M. Alfano, G.L. Blackburn, P. S. Rabinovitch, A. McTiernan, Independent and combined effects of dietary weight loss and exercise on leukocyte telomere length in postmenopausal women, Obesity 21 (2013) E549-E554.

[43] K.A. Mather, A.F. Jorm, R.A. Parslow, H. Christensen, Is telomere length a biomarker of aging? A review, J. Gerontol. A Biol. Sci. Med. Sci. 66 (2011) 202-213.

[44] M. Nyberg, S.P. Mortensen, H. Cabo, et al., Roles of sedentary aging and lifelong physical activity in exchange of glutathione across exercising human skeletal muscle, Free Radic. Biol. Med. 73 (2014) 166-173.

[45] S. Oesen, B. Halper, M. Hofmann, W. Jandrasits, B. Franzke, E.M. Strasser, A. Graf, H. Tschan, N. Bachl, M. Quittan, K.H. Wagner, B. Wessner, Effects of elastic band resistance training and nutritional supplementation on physical performance of institutionalised elderly - a randomized controlled trial, Exp. Gerontol. 72 (2015) 99-108, http://dx.doi.org/10.1016/j.exger.2015.08.013.

[46] D. Ornish, J. Lin, J. Daubenmier, et al., Increased telomerase activity and comprehensive lifestyle changes: a pilot study, Lancet Oncol. 9 (2008) 1048-1057.

[47] M. Pittaluga A. Sgadari, I.Dimauro, B.Tavazzi, P. Parisi, D.Caporossi, Physical exercise and redox balance in type 2 diabetics: effects of moderate training on biomarkers of oxidative stress and DNA damage evaluated through comet assay, Oxid. Med. Cell Longev, 2015:981242.

[48] W. Palm, T. de Lange, How shelterin protects mammalian telomeres, Annu. Rev. Genet. 42 (2008) 301-334.

[49] Z. Radak, A.W. Taylor, M. Sasvari, et al., Telomerase activity is not altered by regular strenuous exercise in skeletal muscle or by sarcoma in liver of rats, Redox Rep. 6 (2001) 99-103.

[50] M. Rantakokko, M. Manty, T. Rantanen, Mobility decline in old age, Exerc Sports Sci. Rev. 41 (2013) 19-25.

[51] N.D. Reeves, M.V. Narici, C.N. Maganaris, Musculoskeletal adaptations to resistance training in old age, Man Ther. 11 (2006) 192-196.

[52] P.J. Robson-Ansley, A. Saini, C. Toms, et al., Dynamic changes in dna methylation status in peripheral blood mononuclear cells following an acute bout of exercise: potential impact of exercise-induced elevations in interleukin-6 concentration, J. Biol. Regul. Homeost. Agents 28 (2014) 407-417.

[53] T. Rönn, P. Volkov, C. Davegårdh, T. Dayeh, E. Hall, A.H. Olsson, E. Nilsson, A. Tornberg, M. Dekker Nitert, K.F. Eriksson, H.A. Jones, L. Groop, C. Ling, A six months exercise intervention influences the genome-wide DNA methylation pattern in human adipose tissue, PLoS Genet. 9 (2013) e1003572.

[54] J. Rutanen, N. Yaluri, S. Modi, et al., SIRT1 mRNA expression may be associated with energy expenditure and insulin sensitivity, Diabetes 59 (2010) 829-835.

[55] N. Sallam, I. Laher, Exercise modulates oxidative stress and inflammation in aging and cardiovascular diseases, Oxid. Med. Cell Longev, vol. 2016, 2016, p. 7239639.

[56] G. Saretzki, Z.T. Von Zglinicki, Replicative aging, telomeres, and oxidative stress, Ann. N. Y. Acad. Sci. 959 (2002) 24-29.

[57] S. Savela, O. Saijonmaa, T.E. Strandberg, et al., Physical activity in midlife and telomere length measured in old age, Exp. Gerontol. 48 (2013) 81-84.

[58] R.K. Schindhelm, L.P. van der Zwan, T. Teerlink, P.G. Scheffer, Myeloperoxidase: a useful biomarker for cardiovascular disease risk stratification? Clin. Chem. 55 (2009) 1462-1470.

[59] R.A. Shanely, D.C. Nieman, D.A. Henson, F. Jin, A.M. Knab, W. Sha, Inflammation and oxidative stress are lower in physically fit and active adults, Scand. J. Med. Sci. Sports 23 (2013) 215-223.

[60] R.J. Shepard, P.N. Shek, Impact of physical activity and sport on the immune system, Rev. Environ. Health 11 (1996) 133-147.

[61] E. Sillanpää, T. Törmäkangas, T. Rantanen, J. Kaprio, S. Sipilä, Does telomere length predict decline in physical functioning in older twin sisters during an 11 -year follow-up? Age 38 (2016) 34

[62] L.C. Silva, A.L. de Araújo, J.R. Fernandes, S. Matias Mde, P.R. Silva, A.J. Duarte, L. E. Garcez Leme, G. Benard, Moderate and intense exercise lifestyles attenuate the effects of aging on telomere length and the survival and composition of $\mathrm{T}$ cell subpopulations, Age 38 (2016) 24.

[63] J.M.1 Starr, P.G. Shiels, S.E. Harris, A. Pattie, M.S. Pearce, C.L. Relton, I.J. Deary, Oxidative stress, telomere length and biomarkers of physical aging in a cohort aged 79 years from the 1932 Scottish Mental Survey, Mech. Ageing Dev. 129 (2008) 745-751, http://dx.doi.org/10.1016/j.mad.2008.09.020.

[64] Q.A. Sun, Y. Wu, F. Zappacosta, et al., Redox regulation of cell signaling by selenocysteine in mammalian thioredoxin reductases, J. Biol. Chem. 274 (1999) 24522-24530.

[65] M. Suwa, K. Sakuma, The potential role of sirtuins regarding the effects of exercise on aging- related diseases, Curr. Aging Sci. 6 (2013) 178-188.

[66] U. Svenson, K. Nordfjäll, D. Baird, et al., Blood cell telomere length is a dynamic feature, PLoS One 6 (2011) e21485.

[67] G. Voghel, N. Thorin-Trescases, N. Farhat, et al., Cellular senescence in 
endothelial cells from atherosclerotic patients is accelerated by oxidative stress associated with cardiovascular risk factors, Mech. Ageing Dev. 128 (2007) 662-671.

[68] T. von Zglinicki, Oxidative stress shortens telomeres, Trends Biochem. Sci. 27 (2002) 339-344.

[69] L.E. Voorrips, A.C. Ravelli, P.C. Dongelmans, P. Deurenberg, W.A. Van Staveren, A physical activity questionnaire for the elderly, Med. Sci. Sports Exerc. 23 (1991) 974-979.

[70] D.E. Warburton, C.W. Nicol, S.S. Bredin, Health benefits of physical activity: the evidence, CMAJ 174 (2006) 801-809.

[71] C. Werner, T. Furster, T. Widmann, et al., Physical exercise prevents cellular senescence in circulating leukocytes and in the vessel wall, Circulation 120 (2009) 2438-2447.
[72] W.R. Wilson, K.E. Herbert, Y. Mistry, et al., Blood leukocyte telomere DNA content predicts vascular telomere DNA content in humans with and without vascular disease, Eur. Heart J. 29 (2008) 2689-2694.

[73] J. Woo, R. Yu, N. Tang, J. Leung, Telomere length is associated with decline in grip strength in older persons aged 65 years and over, Age 36 (2014) 9711 http://dx.doi.org/10.1007/s11357-014-9711-7.

[74] I.N. Zelko, T.J. Mariani, R.J. Folz, Superoxide dismutase multigene family: a comparison of the CuZn-SOD (SOD1), Mn-SOD (SOD2), and EC-SOD (SOD3) gene structures evolution, and expression, Free Radic. Biol. Med. 33 (2002) $337-349$.

[75] F.F. Zhang, R. Cardarelli, J. Carroll, et al., Physical activity and global genomic DNA methylation in a cancer-free population, Epigenetics 6 (2011) 293-299. 